\title{
GUÍA (BREVE) PARA LA INVESTIGACIÓN Y ESTUDIO DE LA LITERATURA ESPAÑOLA: DOCUMENTACIÓN Y BIBLIOGRAFÍA EN INTERNET
}

JAVIER SAlazar Rincón UNED. La Seu d'Urgell

\section{REsumen}

El presente artículo pretende of recer una orientación básica acerca de las principales fuentes de información y documentación disponibles en páginas de Internet en el momento actual para el estudio e investigación de la literatura española, con el convencimiento de que en un plazo muy breve, y por fortuna, algunos de los datos que ofrecemos a continuación quedarán sin duda alguna anticuados, dada la velocidad con que se renueva y actualiza la información que la red ofrece al usuario, y, en nuestro caso, al profesor, al investigador y al estudiante. No obstante, en las páginas que siguen podrán encontrarse algunas direcciones y recursos, que esperamos sean útiles al menos por el momento, relativos a bibliotecas, archivos, seminarios y centros de investigación, bases de datos bibliográficas, revistas electrónicas, bibliotecas virtuales, colecciones de textos, o librerías y editoriales especializadas en el campo de las humanidades.

Junto a los sistemas de documentación tradicionalmente empleados en la investigación literaria para la búsqueda y recopilación de fuentes, bibliografía y documentos impresos (ficheros y catálogos de archivos y bibliotecas, reper- 
torios bibliográficos o revistas especializadas), la extensión creciente y la popularización de los medios informáticos, y particularmente de Internet, han hecho surgir nuevos sistemas y vías de información que, sin desbancar del todo a los recursos clásicos citados, y más bien como complemento de ellos, han variado la metodología tradicional de la investigación en ciencias humanas, creando nuevas posibilidades que por ahora no han hecho más que vislumbrarse, y que a la larga serán revolucionarias.

Como se sabe, Internet es una red en la que se conectan entre sí múltiples ordenadores y redes de ordenadores, que utilizan un protocolo propio y específico para el intercambio de información en todo el mundo, y que proporciona al usuario varios servicios y posibilidades, entre los que destacaremos los siguientes:

1. El acceso a la información contenida en las llamadas páginas Web, en que podemos hallar imágenes, textos escritos, videos, registros sonoros, o directorios y listas muy variadas, con el fin de consultarlas, y, en caso de que nos interese su conservación, imprimirlas en papel o almacenarlas en el disco duro de nuestro ordenador o en un disquete. Muchas de estas páginas nos ofrecen además la posibilidad de conectarnos a otras relacionadas con ellas.

2. El acceso a grandes ordenadores, los de las bibliotecas y centros de documentación, por ejemplo, la ejecución interactiva y a distancia de los programas contenidos en ellos, y la recuperación de la información que almacenan.

3. La captación de la información reunida en los ficheros de un ordenador remoto y su traslado a nuestro ordenador, con el fin de almacenarla en nuestro disco duro o en un disco portátil.

4. El correo electrónico, que nos permite enviar y recibir mensajes escritos, los cuales pueden ir a su vez acompañados de ficheros de texto, imágenes o sonido.

5. Tertulias y grupos de discusión, también llamados chat, en que participan, intercambiando mensajes escritos de manera simultánea, personas unidas por intereses afines.

No entraremos a examinar con detalle cada uno de los posibles usos de Internet que acabamos de citar, ni su funcionamiento en la práctica, ya que sobre ellos existe una bibliografía abundante y asequible a cualquier usuario interesado, así como numerosos cursos organizados por entidades públicas y privadas, limitándonos en las páginas que siguen a comentar las posibilidades que Internet ofrece a quien pretende desarrollar una labor de investigación en el campo de las ciencias humanas, y particularmente en el de la literatura española. 
A través de Internet, el investigador puede intercambiar información con personas que trabajan en su misma área a través del correo electrónico, solicitar el envío de información por el mismo medio, o de libros, fotocopias o documentos impresos a través del correo ordinario, o bien participar en los grupos de discusión o chats antes nombrados, aunque en el trabajo de documentación serán sin duda los tres primeros servicios de Internet que hemos citado los que principalmente interesarán al estudioso.

En efecto, Internet nos permite consultar a distancia los ficheros de las bibliotecas o archivos, acceder a bases de datos bibliográficas o documentales y buscar en ellas información específica, consultar otras fuentes de interés, como los índices de las revistas, los catálogos de las editoriales y librerías privadas, las listas de documentos o de bibliografía elaboradas por instituciones educativas, de investigación o por particulares, e incluso leer documentos escritos, a veces obras literarias o estudios completos, que se encuentren disponibles en la red. En todos estos casos, con el fin de ahorrar trabajo, lo más recomendable es la impresión en papel de dichas páginas o ficheros, si no son muy extensos, o bien su traslado al disco duro de nuestro ordenador o a un disco portátil. Ello nos permitirá su consulta cómoda y rápida, evitándonos la a menudo enojosa tarea de volver a conectamos a la red para su lectura. Conviene sin embargo recordar que muchas de esas páginas y bases de datos documentales están renovándose de manera constante, por lo que conviene volver a ellas de manera periódica con el fin de evitar que la información quede anticuada.

Antes de pasar a comentar algunas direcciones de Internet que pueden resultar especialmente útiles para la investigación literaria, convendrá que recordemos algunas cuestiones que habrá que considerar al iniciar nuestro trabajo de búsqueda, y que deberemos tener claras antes de conectamos a Internet, con el fin de ahorrar esfuerzo, tiempo y también dinero:

La primera de ellas se refiere al campo en que se va a desarrollar nuestra investigación, y podría formularse así: Antes de saber dónde debemos buscar la información, es preciso tener claro qué tema queremos investigar, y por consiguiente, qué registros bibliográficos o documentales interesa consultar. Deslindar con exactitud nuestro tema de investigación, su contenido y sus límites, es una tarea imprescindible, previa a cualquier otra.

La segunda cuestión, directamente derivada de la anterior, consistirá en saber, aunque sea de manera aproximada, qué tipo de documentos nos interesará localizar: obras de un autor, período o movimiento concretos, documentos históricos coetáneos, es decir, fuentes primarias, o bien libros y artículos relacionados con ellos.

La tercera y última cuestión que debemos tener clara antes de sentamos ante el ordenador e iniciar nuestro trabajo de búsqueda, consistirá en conocer 
los criterios de esa búsqueda, y los campos de la base de datos que estemos consultando en que deberemos operar. Puede ocurrir, en efecto, que la dirección de Internet a la que accedamos sea una lista de obras alfabética, o a lo sumo clasificada por temas, en cuyo caso deberemos armarnos de paciencia y leerla entera después de imprimirla o trasladarla a un disquete. En la mayoría de los casos, sin embargo, especialmente en los catálogos de las grandes bibliotecas y centros de documentación, el usuario debe señalar lo que está buscando, especificando con claridad ciertos datos, bien escribiendo una palabra o una frase en un solo campo (búsqueda simple), bien señalando simultáneamente varios datos en uno o más campos (búsqueda avanzada o combinada).

También puede ocurrir que la base de datos que consultemos permita la utilización de comodines, como el asterisco (*) o el signo de interrogación (?), lo cual facilitará y ampliará nuestra búsqueda, de manera que para localizar bibliografía relacionada con el Romanticismo, por ejemplo, bastará con que escribamos «Romant*», en el campo relativo al título, o en el de tema o materia, con lo que la respuesta que obtengamos incluirá todas aquellas obras en que aparezcan las palabras «Romanticismo», «Romantisme», «Romanticisme» «romántico» «románticos», "romántica», «romantic» etc. En otros casos, sobre todo en grandes bases de datos, será necesario recurrir a los llamados operadores booleanos, comunes en el lenguaje informático, y entre los cuales son los más comunes AND, OR, NEAR, NOT. El primero de ellos (AND) indica la intersección de dos conjuntos, y nos proporcionará todos aquellos textos o ficheros en que los datos que hemos escrito se den juntos: así, la frase «Literatura AND española AND medieval» permite recuperar las cadenas, los registros de una biblioteca por ejemplo, en que las tres palabras aparezcan a la vez. OR indica la unión de dos conjuntos, y muestra los textos en que las palabras que pedimos aparecen unidas o por separado: «Cervantes OR Quijote» recupera los ficheros en que aparecen ambas palabras (Cervantes + Quijote), y también las que sólo contienen una de ellas. La expresión NEAR indica proximidad, y buscará aquellos textos en que dos términos aparezcan separados por diez palabras o menos; y NOT indica exclusión, de manera que nos permite eliminar un subconjunto dentro de un conjunto más amplio: así, al ejecutar la orden «Literatura NOT inglesa», el programa buscará todas aquellas cadenas en que aparezca la palabra «literatura», exceptuando aquellas en que vaya acompañada por el adjetivo «inglesa».

Los campos en que podemos buscar dentro de las bases de datos bibliográficas accesibles a través de Internet, coinciden generalmente con los datos básicos que figuran en las fichas de las bibliotecas, y suelen ser los siguientes:

1. Autor: Será el criterio dominante si buscamos un libro concreto, cuyo autor conocemos, o bien todas las obras de un autor determinado: el 
poeta, dramaturgo o novelista sobre el que versa el trabajo (Cervantes, Lorca, García Márquez); o bien un investigador del cual nos consta que es una autoridad en la materia: Ramón Menéndez Pidal, Colin Smith y Edmund de Chasca, por ejemplo, si estudiamos el Poema del Cid.

2. Título: Puede tratarse de un título concreto, en cuyo caso lo escribiremos completo, $o$ indicando al menos sus palabras principales, o bien los títulos referentes a un autor, una época, un tema o un método, en cuyo caso escribiremos ciertas palabras clave, completas o con comodines, que suponemos figurarán en los títulos que nos interesan: así Renac*, Romant*, Cervantes, Góngora, armas, letras, amor, estilística, semio*, etc.

3. Ciudad: No suele ser un criterio importante, aunque en ocasiones se podrá tener en cuenta.

4. Editorial y/o colección: Nos permitiría acceder a los títulos publicados por una determinada editorial, universidad o centro de investigación, o incluidos en colecciones especializadas en alguna disciplina.

5. Año $o$ años: Ya que en ocasiones podemos establecer un año inicial y otro final para efectuar la búsqueda, suponiendo que nos interese localizar únicamente las obras publicadas durante un período determinado: las posteriores a 1990 , por ejemplo, si las anteriores a esa fecha ya las hemos conocido a través de un manual o repertorio impreso.

6. $C D U$ : Los números de la Clasificación Decimal Universal, que asignan a cada área de conocimiento un número específico, pueden ser otro instrumento de búsqueda interesante, en cuyo caso, si no disponemos de la tabla completa de CDU, que publica y renueva periódicamente la Asociación Española de Normalización y Certificación (AENOR), bastará con localizar un título de los que nos interesen, anotar su número, y utilizarlo posteriormente para buscar nuevos títulos que versen sobre el mismo tema.

7. Tema o materia: Es uno de los campos fundamentales para la investigación bibliográfica, ya que, si accedemos a él con criterios adecuados, puede proporcionamos la lista completa de los títulos relacionados con un tema, disponibles en una biblioteca o base de datos. El trabajo de búsqueda se convierte, sin embargo, en una tarea estéril o engorrosa si no sabemos con certeza la palabra o palabras clave que debemos usar para localizar información. Para solucionarlo podemos acudir a la lista de encabezamiento de materias común a las bibliotecas españolas, o, como en el caso anterior, anotar los términos que aparecen en alguno de los títulos que hayamos localizado, y aprovecharlos en búsquedas posteriores. En algunos casos, así ocurre en el 
catálogo automatizado de las principales bibliotecas, pulsando sobre el campo de tema o materia correspondiente a un libro cualquiera, que aparece resaltado en la pantalla, podemos acceder a los demás títulos pertenecientes a ese mismo campo.

$\mathrm{Y}$ con ello pasamos ya a examinar de manera ordenada algunas de las direcciones cuya consulta nos parece imprescindible en la primera fase de todo trabajo de investigación en humanidades, en nuestro caso la literatura: el de la recopilación de las fuentes y bibliografía; y empezaremos, como es lógico, por las bibliotecas españolas generales y especializadas en que podemos localizar dicha información.

La Biblioteca Nacional de Madrid, con más de cinco millones de volúmenes conservados en su fondo, es la primera entre las bibliotecas españolas, puesto que posee, junto a un importantísimo archivo de manuscritos, grabados y libros antiguos, todas las obras publicadas en España desde su fundación, en 1712, que ingresan en ella a través del Depósito Legal, así como muchas otras obras y estudios de interés general para el hispanista publicadas fuera de nuestro país. Como se sabe, entre los tesoros de la Biblioteca figuran el único códice conservado del Poema de Mio Cid, uno de los tres códices del Libro de buen amor, el manuscrito de las Rimas escrito por el propio Bécquer, titulado Libro de los gorriones, y una importantísima colección cervantina, con ediciones, manuscritos y estudios relativos al autor del Quijote de más de 15.000 documentos.

La página de Internet de la Biblioteca Nacional (www.bne.es) da acceso a sus catálogos, que pueden consultarse a través del propio programa de búsqueda creado por la Biblioteca (ARIADNA), y visualizarse después en la pantalla a través de ARIADNA, del ISBD, sistema normalizado para mostrar documentos bibliográficos, o mediante IBERMARC, que exhibe los campos y subcampos utilizados en la catalogación. Para la consulta pueden introducirse datos relativos al autor, nombre de congreso o entidad; título; colección; tema o materia; lugar de publicación; editorial; ISBN; fecha de publicación, indicando año concreto, o dos años entre los que establecemos los límites de la búsqueda. Una vez obtenido uno de los registros, presionando con el ratón sobre el nombre, podemos acceder a la lista completa de las obras de ese mismo autor, o, lo que es más importante, a otras que figuran recogidas en el mismo campo bajo el epígrafe de tema o materia. Desgraciadamente, a pesar del enorme avance que ha experimentado la informatización de la Biblioteca, aún no han sido introducidos en el catálogo automatizado todos sus fondos, por lo que durante algún tiempo seguirá siendo necesario acudir a los catálogos manuales para conocer muchas obras anteriores a 1978. En fin, la página Web de la Biblioteca Nacio- 
nal también da acceso a otros muchos servicios útiles, como el catálogo de publicaciones de la Biblioteca, el Catálogo Colectivo de Publicaciones Periódicas Españolas o el Directorio de bibliotecas y editoriales españolas.

La Biblioteca de Catalunya es otro interesante centro de documentación e investigación, ya que cumple en parte las funciones de biblioteca nacional dentro de su respectivo ámbito, y recoge, a través del depósito legal, un ejemplar de cada obra impresa en dicha comunidad. Fue fundada en 1907, y en la actualidad posee un fondo de más de un millón de documentos. Como dato de interés señalaremos que la Biblioteca de Catalunya posee la segunda colección cervantina más importante del mundo, con más de 9.000 obras de Cervantes, traducciones y estudios relativos al autor.

La página Web de la Biblioteca de Catalunya (www.gencat.es/bc) da acceso a la información general sobre la institución, y al llamado catálogo en línea para la consulta de sus fondos, el cual incluye a su vez las opciones de búsqueda simple, combinación de palabras y búsqueda experta. Sin embargo, igual que ocurre en la Biblioteca Nacional, el antiguo catálogo manual de la Biblioteca de Catalunya no ha sido incorporado por completo a su base de datos automatizada, aunque sí puede consultarse en Internet la reproducción fotográfica de todas sus papeletas, a las que accedemos por orden alfabético. La página de la Biblioteca ofrece así mismo la posibilidad de conocer el Catálogo Colectivo del Patrimonio Bibliográfico de Cataluña, que recoge más de 50.000 registros de obras del siglo XIX y anteriores, correspondientes a diversas bibliotecas catalanas, con indicación de su localización.

Otras bibliotecas públicas españolas no universitarias de importancia, a cuyos catálogos automatizados podemos acceder a través de Internet, y que cuentan a su vez con pasajes para entrar en otras instituciones, bibliotecas y páginas especializadas, son la Biblioteca de Castilla y León (www.bcl.jcyl.es), la Biblioteca Pública de Valladolid (bpval.bcl.jcyl.es), la Biblioteca de Andalucía (www.sba.junta-andalucía.es), la Biblioteca de Aragón e Instituto Bibliográfico Aragonés(www.aragob.es/bibara/pagiba/prima.htm) y la Biblioteca Pública de Valencia (www.gva.es/bpv).

Conviene sobre todo tener presente que, para el acceso a las bibliotecas públicas españolas, cuyos catálogos se hallan informatizados en la mayoría de los casos, contamos con dos importantes instrumentos de información y búsqueda proporcionados por el Ministerio de Cultura, a través de cuya página principal (www.mcu.es) podemos consultar el directorio de las bibliotecas públicas del Estado (www.mcu.es/bp/info.html), así como los ficheros automatizados de cada una por separado, y lo que es más útil, la base de datos en que se hallan incluidos los catálogos de todas ellas (www.mcu.es/bpe/bpe.html), y en la cual disponemos de un programa que permite introducir datos de bús- 
queda en los campos siguientes: General, autor, título, materia, lugar de publicación, editorial, año de publicación y tipo de documento. El resultado nos dará la lista completa de las bibliotecas en que puede hallarse el libro o libros que nos interesen, y la vía para acceder a ellas. A través de la Web del Ministerio de Educación y Cultura, o bien directamente, podemos acceder asimismo a la Red de Bibliotecas del Instituto Cervantes, RBIC (www.cervantes.es/rbic.ht$\mathrm{ml}$ ), y al catálogo colectivo de sus fondos, repartidos por todo el mundo. La misma institución cuenta con el llamado Centro Virtual Cervantes (cvc.cervantes.es), que nos proporciona información y vínculos para acceder a diversas bibliotecas, bases de datos, diccionarios, librerías, editoriales, textos electrónicos, y a otros contenidos de carácter cultural referentes a España e Hispanoamérica.

Fundamentales para el investigador son los fondos bibliográficos del Consejo Superior de Investigaciones Científicas, desde una de cuyas páginas (www.csic.es/cbic/cbic.htm) podemos acceder a las distintas bibliotecas dependientes de esta institución. Entre ellas destacan, sobre todo, para la investigación en el campo de la literatura española, la biblioteca central del CSIC (www.csic.es/cbic/central/central.htm), la biblioteca del Instituto de Filología (www.filol.csic.es/biblioteca/biblioteca.htm), así como la página en que se nos proporciona la relación de todas las bibliotecas del Consejo dedicadas a humanidades y ciencias sociales (www.csic.es/cbic/huma.htm), con el acceso a sus correspondientes páginas de información. Los catálogos de las bibliotecas dependientes de CSIC se encuentran además reunidos en una base de datos única, denominada CIRBIC (sauco.csic.es: 4500/ALEPH/SPA/MAD.LIB/ FILE/base-info), lo cual nos permite acceder con comodidad a todos sus fondos. Dicha base de datos incluye más de 700.000 registros, entre libros, publicaciones periódicas, mapas y documentos iconográficos, y en ella puede consultarse, bien una lista alfabética de obras, o bien una selección de títulos obtenida mediante la introducción de ciertos datos en los campos siguientes: autor, título, materia, número de registro, CDU e ISBN. Una vez hemos accedido a uno de los registros, podemos obtener otros correspondientes al mismo autor, materia o tema.

Los catálogos de las bibliotecas universitarias españolas, cuyas características no nos detendremos a enumerar en este trabajo, también se hallan disponibles en la red, y el acceso a ellos resultará muy sencillo: El Ministerio de Educación y Cultura ofrece un directorio desde el cual podemos trasladamos a la página inicial de todas las universidades españolas (www.mec.es/recursos/centros/univ.es.html), y desde allí, a sus respectivas bibliotecas. Las bibliotecas universitarias y centros de investigación españoles se encuentran además coordinados a través de la Red Iris (Red Académica y de Investigación 
Española), cuyas páginas (www.rediris.es/recursos/bibliotecas) nos facilitan la entrada tanto en las bibliotecas universitarias, como en las del Consejo Superior de Investigaciones Científicas. El propio CSIC cuenta, por su parte, con un servicio similar (www.csic.es/cbic/webuni.htm), a través del cual podemos llegar hasta las principales bibliotecas universitarias, las cuales se hallan además coordinadas a través de la Red de Bibliotecas Universitarias Españolas, REBIUN, en cuya página Web (www2.uji.es/rebiun/), podemos consultar directorios, información sobre actividades, proyectos y catálogos. Varias de estas bibliotecas, doce en total, entre ellas la de la UNED, que utilizan el sistema de gestión bibliotecaria DOBIS/LIBIS, se encuentran coordinadas a través del programa RUEDO (Red Universitaria Española Dobis / Libis), desde cuya página Web, con sede en Oviedo (buo.uniovi.es/Buo-Ruedo.html), podemos acceder a diversos servicios, y a su catálogo colectivo, con más de dos millones de registros bibliográficos. Otros consorcios que agrupan a diversas universidades, y a través de cuyas páginas podemos acceder cómodamente al catálogo colectivo de sus bibliotecas, son la organización RUECA, Red Universitaria Española Absys (www.baratz.es/cgi.bin/abwebr), que coordina a las universidades que utilizan dicho sistema de catalogación; y el Consorci de Biblioteques Universitàries de Catalunya (www.cbue.es), integrado por los centros universitarios de dicha comunidad. La Asociación de Editoriales Universitarias Españolas cuenta además con una página propia (www.acue.es), en que se incluye el catálogo colectivo de sus publicaciones.

Otras instituciones educativas y de investigación, además del NEC y el CSIC, también nos proporcionan completos directorios de bibliotecas españolas, tanto generales como universitarias: Así, el Departamento de Biblioteconomía y Documentación de la Universidad de León (www.unileon.es/dp/dph/ recursos/bibdoc/index.htm) ha diseñado una página que da acceso a los servidores y catálogos de bibliotecas universitarias españolas, al tiempo que presenta un directorio y completa información sobre bases de datos, centros de investigación y bibliotecas públicas españolas y extranjeras. Un trabajo similar de recopilación de direcciones sobre bibliografía, biblioteconomía y documentación, con información acerca de bibliotecas de todo el mundo, ha sido elaborado por la Biblioteca de la Universidad Complutense de Madrid (www.ucm.es/BUCM. Directorios parecidos, disponibles en la red, son el mapa de recursos españoles en Internet de la Universidad de Oviedo (index.uniovi.es/Recursos), el directorio de bibliotecas españolas elaborado por la Biblioteca Pública del Estado de Valladolid (bpval.bcl.jcyl.es/OtrasBibliotecas. shtml), o el directorio de recursos bibliotecarios y accesos a bibliotecas españolas preparado por la Universidad de Valencia (www.uv.es/biblios/webs/ web-Espa.html). Muy útil resultará la consulta de la sección titulada Recursos 
de Internet, elaborada por la Biblioteca de la Universidad Nacional de Educación a Distancia (info.uned.es/biblioteca/enlaces.htm), en la que se incluyen directorios jerarquizados de enlaces, listas de bibliotecas, bases de datos y publicaciones periódicas. Para los estudios de filología en sus diversas especialidades, resulta especialmente recomendable la completa colección de direcciones y vínculos reunida por la Biblioteca de la Universidad de Barcelona bajo el título Fent Filologia per Internet (www.bib.ub.es/www8/ Orec.htm).

La información sobre los archivos estatales españoles, e incluso la consulta de sus fondos, nos la facilita el Ministerio de Educación y Cultura a través de una página (www.mcu.es/lab/archivos), en que es posible encontrar datos acerca de los archivos dependientes directamente del MEC y otros gestionados por los gobiernos autónomos, un directorio de archivos iberoamericanos, un formulario para buscar documentos en las bases de datos de los archivos españoles cuyos catálogos se encuentren informatizados en la actualidad, y otro para la búsqueda de bibliografía relativa a archivos. Entre todos ellos destacan, como sabemos, por su importancia para el investigador, el Archivo Histórico Nacional, el Archivo de Simancas, el Archivo de Indias y el Archivo de la Corona de Aragón.

Varias bases de datos bibliográficas españolas resultarán útiles para la búsqueda de información de carácter general, o relativa a una especialidad en concreto, y entre ellas mencionaremos en primer lugar la que mantiene la Agencia Española del ISBN, dependiente del Ministerio de Cultura (www.mcu.es/bases/spa/isbn/ISBN.html), que se actualiza anualmente, y en la que se incluyen los datos esenciales de todos los libros editados en España desde 1966. En ella podemos buscar obras introduciendo datos en los campos siguientes: autor, título, año, editorial, lengua, ISBN. El propio Ministerio de Cultura, en colaboración con las comunidades autónomas, nos proporciona el acceso al Catálogo Colectivo del Patrimonio Bibliográfico Español (www.mcu.es/ccpb/index.html), importante base de datos, con la descripción de cerca de 300.000 obras impresas entre los siglos XV y XIX, y al catálogo automatizado de los más de medio millón de ejemplares de las mismas depositados en unas 500 bibliotecas públicas y privadas. Junto a ella, la base de datos denominada REBECA (www.mcu.es/rebeca), también del Ministerio de Cultura, con más de 300.000 registros, está concebida para facilitar a las bibliotecas la catalogación de sus fondos, y puede resultar igualmente útil para el investigador: entre otras cosas incluye las referencias de cerca de 20.000 artículos de revistas culturales. El Consejo Superior de Investigaciones Científicas nos proporciona a través de Internet el acceso a uno de sus organismos, el Centro de Información y Documentación Científica, CINDOC (www.cindoc.csic.es/prod/dbsconx. html), el cual ofrece a su vez la posibilidad de con- 
sultar varias bases de datos especializadas, en que se recogen más de medio millón de referencias bibliográficas correspondientes a más de dos mil revistas profesionales. Entre estas bases de datos nos interesará especialmente la denominada ISOC, dedicada a ciencias sociales y humanidades. Los datos referentes a las tesis doctorales leídas en universidades españolas a partir de 1976, también se encuentran disponibles en una base de datos denominada TESEO, del Ministerio de Educación y Cultura (www.mec.es/teseo), en la cual podemos realizar una búsqueda, simple o combinada, dentro de los campos siguientes: Título y resumen, descriptores, autor, director, curso y universidad.

El estudio, la edición y la interpretación de los textos literarios topa, de otro lado, con problemas semánticos, léxicos y críticos que los investigadores solemos resolver acudiendo a los vocabularios ya publicados de un autor o de un período, o mediante la consulta de diccionarios antiguos como el de Covarrubias o el de Autoridades, otros modernos como el de María Moliner y el reciente Diccionario del español actual de Manuel Seco, o los que periódicamente publica la Academia. Actualmente la Real Academia Española tiene abierta una página Web (www.rae.es) en que se puede encontrar información acerca de esta institución, sus actividades y publicaciones, y consultar su primer diccionario, el llamado de Autoridades (1726-1739), y la última edición del diccionario usual (1992), junto a las enmiendas más recientes aprobadas por dicha corporación. Además de estos instrumentos, la Academia ha puesto a disposición del usuario dos interesantes bancos de datos léxicos (www.rae.es/NIVEL1/ACABANCO.HTM), a los que se puede acceder directamente en su versión restringida, o solicitando una clave de acceso, si se trata de investigadores que pretendan consultar la versión completa. La primera de dichas bases de datos (CREA) tiene carácter sincrónico, y reúne las principales variantes de cada palabra en los países en que el español es lengua mayoritaria; el segundo, de tipo diacrónico (CORDE), ofrece la evolución y usos de cada palabra a lo largo de su historia, con ejemplos de su utilización en la obra de numerosos autores, lo cual resultará especialmente útil, no sólo para los lingüistas, sino también para los especialistas en literatura.

Las páginas y catálogos automatizados de las bibliotecas nacionales y universitarias de otros países también pueden consultarse a través de Internet. Entre ellas destacaremos la Biblioteca del Congreso de los Estados Unidos (Icweb.loc.gov), que presume de ser, y no le faltan motivos, la más completa del mundo: sus catálogos pueden consultarse en línea a través de varios procedimientos, que van desde la búsqueda de palabras simple (de autor, título, materia, etc.), a sistemas de búsqueda combinada y avanzada, en los que es posible introducir simultáneamente más de un dato, o seleccionar dos o más encabezamientos de materia con el fin de realizar búsquedas cruzadas. La Biblioteca del 
Congreso cuenta además con una sala de temas hispánicos, la Hispanic Reading Room, en cuya página y catálogos (lcweb.loc.gov/rr/hispanic/salahisp.html) podemos hallar información de utilidad para el investigador, recursos acerca de las culturas hispánicas disponibles en la red, y el acceso a los registros bibliográficos de las principales bibliotecas de los países ibéricos e iberoamericanos o a datos referentes a ellos. Junto a la Biblioteca del Congreso, destacaremos en Estados Unidos las páginas elaboradas por la Hispanic Society of America (www.hispanic.society), con sede en Nueva York, que nos informan sobre su biblioteca, actividades y recursos. En fin, interesantes para nuestro campo, y para cualquier trabajo de investigación, son la Biblioteca Británica, que cuenta con una página de información general (www.bl.uk) y otra de acceso a su base de datos bibliográfica (www.blaiseweb.bl.uk), o la $\mathrm{Bi}$ blioteca Nacional de Francia (www.bnf.fr), con un importante fondo de textos antiguos y estudios relacionados con las literaturas hispánicas.

El acceso a la información y los catálogos de las demás bibliotecas nacionales y universitarias extranjeras puede realizarse directamente, o bien a través de cómodos directorios que facilitan la información y la entrada a muchas de ellas. Entre ellos citaremos el programa GABRIEL, Gateway to Europe's $\mathrm{Na}$ tional Libraries (portico.bl.uk/gabriel/), en cuyas páginas se nos proporciona información sobre las distintas bibliotecas nacionales europeas, junto a los enlaces para acceder a todas ellas. Muchas otras entidades ofrecen páginas de información académica general, con listas de bibliotecas, bases de datos, centros de documentación y enlaces para su examen y consulta: así, el Bulletin Board for Libraries de la Universidad de Strathclyde en Escocia (link.bubl.ac. uk:80/lis) presenta una completa recopilación de recursos útiles para la comunidad científica y académica, organizada en campos de acuerdo con el sistema de Clasificación Decimal Universal. El Wisconsin Department of Public Instruction (www.dpi.state.wi.us/) nos presenta otra completa lista, en la que se incluyen motores de búsqueda, directorios e información sobre bibliotecas, bases de datos, páginas de instituciones gubernamentales y acceso a numerosos documentos. La Universidad de Lund (Suecia) ha preparado otra lista (www. ub2.lu.se/lisres.html) con un centenar y medio de direcciones especializadas en biblioteconomía y documentación, así como comentarios y enlaces para cada una de ellas. Igualmente interesante es el programa Hytelnet on the World Wide Web (www.lights.com/hytelnet), que contiene una amplia información sobre recursos bibliotecarios y vínculos con las principales bibliotecas del mundo.

Entre las bases de datos elaboradas fuera de nuestro país, destacaremos la que mantiene en Francia el INST, Institut d'Information Scientifique et Technique (www.inist.fr), con amplia información sobre ciencias sociales y huma- 
nidades, y en la que se incluyen 1.700 .000 registros bibliográficos, incluidos artículos aparecidos en numerosas revistas especializadas de todo el mundo. La empresa británica Chadwyck mantiene mediante suscripción una base de datos (pci.chadwyck.com), con más de diez millones de registros, en la que se encuentran artículos aparecidos en revistas y publicaciones periódicas de todo el mundo desde el siglo XVIII; y en Estados Unidos, la base de datos Uncover (uncweb.carl.org), de la Carl Corporation, de acceso gratuito, incluye datos sobre artículos publicados en 17.000 revistas profesionales, a los que podemos acceder por autor, palabra clave o título de la revista.

Otras entidades y empresas ofrecen bases de datos en las que podemos encontrar unificados los registros de distintas bibliotecas y centros de documentación, lo cual nos permitirá su consulta simultánea: Así, la base de datos de la Blaise Web británica (icarus.bl.uk/nbs/blaise/web-manual.html\#files) incluye alrededor de 17 millones de fichas bibliográficas procedentes de la British Library y de otras bibliotecas generales y especializadas. La RLG, Research Library Group (www.rlg.org), agrupa a diversas entidades dedicadas a la investigación y la enseñanza, y ofrece la posibilidad de acceder, a través de su programa, a 27 millones de títulos catalogados en las principales bibliotecas del mundo, principalmente de países de lengua inglesa.

Un instrumento de consulta muy conocido, ahora disponible en Internet, para el estudio de la literatura en general, y también de la literatura española, es la base de datos bibliográfica elaborada por la Modern Language Association of America (www.mla.org), la MLA International Bibliography (www.mla.org/main-bib-nf.htm), en la que se incluyen más de 50.000 títulos anuales distribuidos en los siguientes campos: Literatura de los países de habla inglesa, literatura de otros países, lingüística, aspectos generales de teoría literaria, literatura en general y folklore. El repertorio, disponible en bibliotecas, puede consultarse en su versión impresa, en CD-Rom, y también mediante la suscripción en Internet a través de dos entidades distribuidoras: La Online Computer Library Center (www.oclc.org) con sede en Dublín, Ohio, y Ovid Technologies de Nueva York (www.ovid.com), dos empresas que ofrecen muchos otros servicios relacionados con la información académica y profesional, y nos proporcionan el acceso a numerosas bases de datos comerciales y de carácter público.

La editorial británica Chadwyck, ya citada, nos ofrece otra interesante base de datos bibliográfica sobre literatura española, elaborada por María del Carmen Simón Palmer, que puede adquirirse en CD-Rom o consultarse mediante suscripción a través del servidor de la editorial (ble.chadwyck. co.uk): su catálogo incluye lo publicado después de 1980, y en tanto no se actualice el Manual de Bibliografía de la Literatura Española de José Simón Díaz (Ma- 
drid, Gredos, 1980), parece un instrumento de consulta muy útil para las universidades, centros de investigación e investigadores individuales. La base de datos, accesible en las principales bibliotecas, ofrece la posibilidad de acceder a sus registros, clasificados con criterios cronológicos, por materias, o bien introduciendo datos relativos al autor, reseñador, revista, palabra clave general, palabra clave de título, o año de publicación.

Varias universidades e instituciones científicas extranjeras ofrecen páginas especializadas en literatura española, con un selecto repertorio de fuentes bibliográficas y recursos de investigación. Entre tales recopilaciones de carácter general pueden citarse la Comparative Literature. A Library Research Guide, de la Pennsylvania State University (www.libraries.psu.crsweb/arts/ humanities/complitbib.html), con un apartado dedicado a las literaturas hispánicas; la Guide to Spanish and Latin American Literature, de la Tulane University (www.tulane.edu/ html/latlit.html), la guía titulada Spanish \& Portuguese Literatures de la Universidad de Maryland (www.lib.umd.edu/ UMCP/MCK/ GUIDES/spanish_literature.html), la Spanish and Portuguese Literature Research Guide, creada por la Bobst Library de la Universidad de Nueva York (www.nyu.edu/library/bobst/research/hum/ibero/spanguid. htm), la Research Guide. Spanish and Latin American Language and Literature, mantenida por la Mugar Memorial Library de la Universidad de Boston (www.bu.edu/library/research-guides/spanish.htm), la Research Guide to Spanish Language and Literature de la Universidad de Western Connecticut (www.wcsu.ctstateu.edu/library/lc-classification.html), la Research Guides. Basic Library Resources for Spanish de la Universidad de Indiana (www.indiana.edu/ librcsd/ libguide/salc/sspa.html), Internet Resources for Hispanists (www.humnet. ucla.edu/cifar/hisplink.html) de la Universidad de Califomia en Los Ángeles, y las más breves Spanish and Latin American Literature. A Selective Bibliography of Sources, de la Universidad de Columbia (www.columbia.edu/cu/libraries/indiv/butlref/split.html), la Library \& Internet Resources for French and Spanish, de la University of South Florida (www.lib.usf.edu/ frank/guides/probes.html), las secciones tituladas Latin American Literature Index y Spanish Language and Literature Index, de la Universidad de Alberta en Canada (www.library.ualberta.ca/library_html/subjects/language), y la Petite Guide de Documentation en Littérature Hispanique de la Universidad de Montreal (www.bib.umontreal.ca/SS/hispgd.htm). Muy útiles son las páginas tituladas Iberian Language \& Literature de la Universidad de Yale (www.library.yale.edu/Internet/iberianlanglit.html), y The Spanish and Portuguese Page at UCSD (gort.ucsd.edu/rsonn/spanish.html), de la Universidad de California en San Diego, con numerosos vínculos para acceder a páginas de Intenet, revistas electrónicas, bases de datos, así como a los catálogos de las biblio- 
tecas de Portugal, España y varias de Estados Unidos especializadas en las lenguas y culturas peninsulares. Un interesante panorama de la crítica literaria mundial, con múltiples vínculos y direcciones de interés, aunque con escasa presencia de la literatura española, nos lo proporciona el proyecto en marcha titulado Online Literary Criticism Collection (www.ipl.org/ref/litcrit) de la Universidad de Michigan.

En la actualidad hay varias revistas electrónicas dedicadas a la literatura española disponibles en la red. En España es imprescindible citar, como fuente de carácter general, la revista Espéculo, de la Facultad de Ciencias de la Información de la Universidad Complutense de Madrid (www.ucm.es/info/especulo): se publica desde 1995, con carácter cuatrimestral, y contiene artículos de teoría y crítica literarias, de filosofía y de cuestiones culturales en general. En ella se incluyen una biblioteca quijotesca, en la que es posible hallar textos de numerosos autores que deben algo a Cervantes, y una Biblioteca Especular de estudios literarios y lingüísticos. El Departamento de Filología Española de la Universidad de Zaragoza publica desde 1996 la revista Interletras. Revista de Crítica y Literatura en Lengua Española (fyl.unizar.es/gcorona/interlet. htm), con artículos, textos, fonoteca e información sobre literatura en Internet. Otra interesante revista electrónica especializada en literaturas hispánicas en general, es la denominada PIEL. Páginas Interdisciplinares de Estudios Literarios (huizen.dss.nl/ jaoskam/), que publican en Holanda Manuel L. Abellán y Jeroen Oskarn desde 1989, y que desde 1997 se encuentra disponible en Internet. Los estudiantes de la George Mason University, en EE.UU., editan la revista titulada Hispanic Cultural Review (mason.gmu.edu/ hor/), en inglés y en español, de contenido variado, aunque relacionado siempre con el mundo hispánico. En la Universidad de Valencia se edita desde 1996 la revista Lemir. Revista de Literatura Española Medieval y del Renacimiento (www.uv.es/ -lemir/Revista.html), dirigida por José Luis Canet y especializada en la producción literaria de dicho período, para cuyo estudio también contamos desde 1998 con Laberinto. An Electronic Journal of Early Modern Hispanic Literature and Culture (www.utsa.edu/academics/cofahlaberinto/welcometext. htm), de la Universidad de Arizona, dedicada a estudios críticos, teóricos e interdisciplinarios sobre la producción literaria española e hispanoamericana comprendida entre 1450 y 1750 . Entre las revistas dedicadas a la literatura española contemporánea citaremos la titulada Abel Martín (www.abelmartin.com/), especializada en Antonio Machado, su vida, su obra y su época.

Pasando ya a examinar algunas direcciones especializadas en un autor, género o período determinado, destacaremos la información que ofrece en Internet el Seminario de Estudios Medievales y Renacentistas, dirigido por Pedro Cátedra en la Universidad de Salamanca, en cuyas páginas (www.gugu.usal. 
es/ eco) podemos encontrar textos, bibliografía e información relativa a sus actividades. Datos similares ofrece el Seminario de Filología Medieval y Renacentista dirigido por Carlos Alvar en Alcalá de Henares (www.alcala. es/filmr). Interesante información y varios enlaces pueden hallarse en el programa dedicado a Literatura Española Medieval y del Renacimiento (Lemir) coordinado por José Luis Canet, de la Universidad de Valencia (www.uv.es/-lemir), que incluye el acceso a la publicación periódica Lemir, a la que nos hemos referido más arriba, a una sección de textos medievales, así como a otras dos tituladas Tirant y Memorabilia, dedicadas a los libros de caballerías y a los libros sapienciales respectivamente. La página denominada The Labyrinth: Resources for Medieval Studies (www.georgetown.edu/labyrinth/labyrinth-home.html), de la Georgetown University, muy completa, presenta una sección dedicada a las literaturas peninsulares. La On-Line Reference Books for Medieval Studies (orb.rhodes.edu) ofrece una exhaustiva lista de vínculos relativos a diversos ámbitos de las literaturas medievales europeas. El Internet Medieval Source Book (www.fordham.edu/halsall/sbook. html), al que también podemos acceder desde la página anterior, ofrece entre sus secciones un Libro de fuentes medievales de Internet. La página PhiloBiblon, o Bibliographical Database of Early Texts Produced in the Iberian Peninsula, de la Universidad de Berkeley (sunsite.berkeley.edu/PhiloBiblon/ phhm.html), da acceso a revistas, bibliotecas o catálogos relacionados con las literatura peninsulares de la Edad Media. Cibertextos Castellanos, de la Duke University (www.duke.edu/web/cibertextos), es un programa que permite buscar y consultar obras correspondientes a este período: en él se halla disponible una sección dedicada a la Celestina, que incluye el texto, concordancias, campos semánticos e ideológicos, refranes y ensayos; y se encuentran en preparación los textos del Poema del Cid y el Libro de buen amor. Alfonso X el Sabio y sus cantigas cuentan con una página propia en la Universidad de Washington (faculty.washington.edu/petersen/alfonso/alfonsox.htm). Para el conocimiento de Juan Ruiz es interesante la colección de carácter divulgativo titulada Caminos del Arcipreste de Hita (arcipreste.alcarria.com). En fin, en nuestra Universidad destacaremos la página que José Manuel Fradejas Rueda ha dedicado a los libros de cetrería medievales (www.uned.es/prof/03412/cetreria).

Para los inicios del Renacimiento puede consultarse la ventana titulada Sonetos del Siglo de Oro (ingber.spanish.sbc.edu/Sonnet/Texts/sonnets. html), creada por Alix Ingber, del Sweet Briar College, con textos de Garcilaso y Boscán, e información complementaria. La Universidad de Santiago de Compostela ha elaborado una página (www.usc.es:8080/quevd) con abundante información sobre Quevedo, y acceso a diversas direcciones relacionadas con su época. 
El Grupo de Investigación del Siglo de Oro (Griso), de la Universidad de Navarra (Griso.cti.unav.es), presenta una variada información general, documentación y bibliografía sobre Calderón, Quevedo, Tirso de Molina y la comedia barroca. La Brown University cuenta con una página especializada en Góngora y la poesía barroca (www.brown.edu/Departments/ HispanieStudies/gongora/indice.htm), que ofrece vínculos de interés, textos y un índice de primeros versos. La Universidad de Arizona, y con ella la Association for Hispanic Classical Theater, ha creado una impresionante base de datos dedicada al teatro del Siglo de Oro (listserv.ccit.arizona.edu/pub/listserv/comedia/index.html), en la que podemos consultar los textos de más de doscientas comedias de Lope de Vega, Cervantes, Tirso de Molina, Ruiz de Alarcón, Calderón o Mira de Amescua. Otra página similar, aunque no tan exhaustiva, es la titulada Teatros de los Siglos de Oro, de la Université du Québec a Trois-Rivières (www.uqtr.uquebec.ca/dlmo/TEATRO/teatro.html), que incluye los textos de una veintena de comedias de diversos autores, herramientas de investigación, información sobre congresos, resúmenes de la revista Criticón, y conexión con otras direcciones relacionadas con el tema. Muy especializadas y completas son las páginas dedicadas a la literatura emblemática del Siglo de Oro, una abierta en la Universidad de la Coruña (rosalia.dc.udc.es/emblemática) por un grupo de investigadores, y otra en la Universidad de las Islas Baleares por Antonio Bernat Vistarini (massanella.uib.es/depart/dfe/Bernat), en que se incluyen vínculos, noticias e información bibliográfica de interés.

Cervantes cuenta con importantes recursos reunidos en el proyecto Cervantes 2001 de la Universidad de Texas (www.csdl.tamu.edu/cervantes), en que podemos hallar, además de vínculos con otras páginas dedicadas al autor, la $\mathrm{Cer}$ vantes Bibliography Online (www.csdl.tamu.edu/cervantes/abc94_2. html), elaborada por Eduardo Urbina, en constante proceso de actualización. El Centro de Estudios Cervantinos de Alcalá de Henares (cervantes.alcala.es/) en colaboración con la Universidad de esta ciudad, ofrece en sus páginas, además de una biografía del autor y otras noticias de interés para los cervantistas, las obras completas de Cervantes, con un motor de búsqueda que permite hallar cualquier palabra en los textos. Fred Jelile, de la Universidad de Indiana, ha puesto a disposición del usuario otra cuidada y manejable versión de las obras completas del autor del Quijote (www.ipfw.indiana.edu/cm1/jehle/ web/cervante.htm). La Cervantes Society of America cuenta con una dirección propia (pom-vms1.pomona. edu/ mmcgaha), y ofrece la consulta gratuita, a través de la dirección de la Universidad de Indiana antes citada (www.ipfw.indiana.edu/cm1/jehle/web/cervantes/bcsalist.htm), de la revista Cervantes, órgano de la Asociación. También puede visitarse la página titulada $H$-Cervantes, con enlaces e información para los especialistas, de la Michigan State University (www.n-net.msu.edu/ cervantes). 
Para el siglo XVIII, aunque dedicada básicamente a autores de lengua inglesa, la Caniegie Mellon University, a través de la página titulada EighteenthCentury Studies (english.www.hss.cmu.edu/18th.html\#s), mantiene una interesante colección de obras del siglo XVIII y comienzos del XIX. Para el Romanticismo español, aunque con noticias relativas a otros ámbitos europeos, resultará útil la página creada por la Universidad Jaime I de Castellón (www1.uji.es/ale/), en que puede hallarse información sobre prosa, poesía y teatro, acceso a páginas dedicadas al Romanticismo de otros países europeos, o a Baudelaire, Goethe, Hugo y Lord Byron, además de las especializadas en Bécquer, Espronceda y Rosalía de Castro. Para la novela realista recomendamos las páginas sobre Leopoldo Alas Clarín creadas por Aquiles Zurita (www.maptel.es/clarin/default.htm), con textos del autor, bibliografía y vínculos de interés; y la página titulada The Pérez Galdós Edition Project, de la Universidad de Sheffield (www.shef.ac.uk/uni/projects/gep), en que hallamos un interesante panorama de actividades, y un proyecto de edición electrónica de las obras del autor.

Para el estudio de la literatura española anterior a la Guerra Civil también contamos con varias direcciones electrónicas, entre ellas la que mantiene la Fundación Juan Ramón Jiménez en Moguer (www.fundacion-jrj.es/), con información bibliográfica, noticias, catálogo de publicaciones y programa de actividades. Un contenido similar presenta la página de la Fundación Ortega y Gasset, con sede en Madrid (www.ortegaygasset.edu/). El Archivo Rafael Cansinos Assens (www.cansinos.com/archivo/) ofrece datos sobre la biografía y obra del autor, bibliografía, álbum fotográfico, textos y documentación. La Fundación Max Aub, de Segorbe, promueve una página (www.maxaub. $\mathbf{c o m} /$ ) con información relativa a premios, cursos, publicaciones y becas de investigación. Especialmente útil es la Web de la Fundación Jorge Guillén (www.fjguillen.es) de Valladolid, en la que, además de hallar abundantes referencias al autor, se nos permite acceder, a través de la página titulada $E l \mathrm{Nu}$ do Gordiano, a bibliotecas, librerías, fondos documentales, índices bibliográficos y otros recursos de interés relacionados con la literatura española en general. En cuanto al conocimiento de la obra de García Lorca, resulta imprescindible la consulta de la página abierta en la Fundación Federico García Lorca (www.garcia-lorca.org), con sede en la Residencia de Estudiantes de Madrid, y que cuenta con la colaboración de CSIC. La Residencia de Estudiantes, con el apoyo de la Fundación Marcelino Botín, la Comisión Europea y el Ministerio de Cultura, también ha impulsado la creación de un gran archivo virtual (www.archivovirtual.org), que funcionará como base de datos y sistema de información compartido por distintas colecciones, archivos y centros de investigación, y en que se catalogarán escritos, epistolario personal, fo- 
tografías y dibujos de autores correspondientes a la llamada Edad de Plata de la cultura española, con un inventario total de cerca de un millón de documentos cuando el proyecto haya concluido.

Entre las páginas relacionadas con los autores de posguerra, destacaremos la que en el Padrón mantiene la Fundación Camilo José Cela (www.celafund.es), con abundante información sobre el autor, y sobre la institución, sus fondos y actividades. La Diputación Foral de Guipúzcoa, por su parte, ofrece una página dedicada a la vida y obra de Gabriel Celaya (www.guipuzkoa.net/kultu$\mathbf{r a} / \mathbf{k m} /$ castella/celaya/), en que se incluye una cronología, bibliografía completa, álbum fotográfico, documentos y poemas.

La obra de varios autores hispanoamericanos, o la bibliografía y actividades relacionadas con ellos, también pueden conocerse a través de Internet: Así, el archivo sobre Borges organizado por la Universidad de Aarhus en Dinamarca (www.hum.aau.dk/Romansk/borges/); un completo corpus de literatura argentina contemporánea creado por la Universidad de Buenos Aires, con textos, entrevistas, bibliografía y reportajes de numerosos autores (opium.q1. fcen.uba.ar/Literatura.html); o la página de la Fundación Pablo Neruda, con datos sobre el autor y bibliografía referente a su obra, creada por la Universidad de Chile (www.uchile.cl/actividades_culturales/premios_nobel/neruda/Pablo_Neruda/html).

Además de las páginas mencionadas hasta ahora, la red también permite la consulta de numerosas bibliotecas electrónicas de libre acceso, algunas de las cuales poseen nutridas colecciones. Entre ellas merece una mención especial la Biblioteca Virtual Miguel de Cervantes (cervantesvirtual.com), creada por la Universidad de Alicante con el apoyo de una conocida entidad financiera, que cuenta ya con un importante corpus, y en la que se pretende poner a disposición del usuario 30.000 obras clásicas, la mayoría en castellano, agrupadas por materias de acuerdo con el sistema de Clasificación Decimal Universal. La Biblioteca Virtual Cervantes también se propone editar trabajos científicos y tesis doctorales, y ofrece un acceso cómodo y rápido a las páginas Web de bibliotecas nacionales, bases de datos, archivos, bibliotecas digitales y revistas electrónicas. Una interesante colección de textos ensayísticos escritos en castellano, desde don Juan Manuel hasta José Ortega y Gasset, nos la ofrece José Luis Gómez Martínez, del Departamento de Lenguas Románicas de la Universidad de Georgia (ensayo. rom.uga. edu). Para la literatura escrita en lengua castellana, también destacaremos el Proyecto Cervantes, del Centro de Enseñanza Técnica y Superior de México (ballena.ens.cetys.mx/servicio.html), en el que se intenta almacenar el mayor número posible de textos escritos originalmente en lengua española, o traducidos a ella, no sólo de tipo literario. 
Entre las bibliotecas de este tipo, con predominio de obras escritas en otras lenguas, citaremos ante todo el Proyecto Gutemberg (www.promo. net/pg/), en constante proceso de ampliación, y que cuenta ya con numerosas obras completas de carácter literario, científico o filosófico. Otra interesante base de datos es Athena (un2sg1.unige.ch/athena/html/athome.html), que ofrece una recopilación de cerca de 10.000 textos, básicamente franceses. La página denominada Alex. A Catalogue of Electronic Texts on the Internet (gopher.lib.ncsu.edu/11/library/stacks/Alex), permite buscar textos de diferentes autores siguiendo varios criterios. La Humanities Text Initiative (www.hti.umich.edu/) de la Universidad de Michigan, ofrece el acceso a numerosas colecciones de textos literarios, fundamentalmente de autores ingleses modernos, de consulta libre. The Book Wire Reading Room (www.bookwire.com/links/readingroom/readingroom.html) también nos facilita la lectura directa de diferentes textos electrónicos de dominio público, que aparecen clasificados por géneros y materias. The Universal Library de la Carnegie Mellon University (www.ul.cs.cmu.edu) publica textos literarios y de humanidades en Internet, desde los clásicos griegos hasta nuestro siglo. The Online Books Page (www.cs.cmu.edu/books.html) presenta un índice con más de 10.000 obras en lengua inglesa, de dominio público. En Electronic Text Center (etext.lib.virginia.edu/), de la Universidad de Virginia, se hallan disponibles más de 45.000 textos literarios, humanísticos o relativos a ciencias sociales, con predominio de los autores de lengua inglesa. En fin, una completa relación de bibliotecas virtuales y empresas especializadas en la publicación de libros electrónicos nos la proporciona la Omni Media Digital Publishing (www.exemplary.net/omnimedia/ links.html).

Varias editoriales españolas nos ofrecen, junto a la información relativa a sus servicios, la posibilidad de acceder a sus catálogos, e incluso adquirir sus publicaciones, a través de la red, lo cual deberá tenerse en cuenta para obtener información actualizada acerca de las últimas novedades en el campo de investigación y estudio que nos interese. Entre todas ellas destacaremos las direcciones de algunas empresas especializadas en el campo de la crítica literaria, la literatura española y los estudios hispánicos en general. Así, las páginas con información, direcciones y catálogos de obras que ofrecen las editoriales Gredos (www.editorialgredos.com), Castalia (www.castalia.es), Cátedra (www.catedra.com), integrada en la página general creada por el Grupo Anaya (www.anaya.es), Península (www.partal.com/ peninsula), Destino (www.globalcom.es/destino), Visor Libros (www.libronet.com/Editor/Visor), Iberoamericana (www.iberoamericana libros.com), Alianza Editorial (www.alianzaeditorial.es) y Círculo de Lectores (www.circulolectores. com). También las universidades, bibliotecas 
nacionales y centros de investigación que hemos citado anteriormente, ofrecen habitualmente a través de la red sus propios catálogo de publicaciones.

En cuanto a las librerías españolas que vienen atendiendo desde hace años a la comunidad científica y universitaria, citaremos los servicios que actualmente ofrecen en la red León Sánchez Cuesta (globalcom.es/saculib), Marcial Pons (www.marcialpons.es), Crisol (www.crisol.es), la Casa del Libro (www.casadellibro.com) y Fuentetaja (www.expocenter.com/fuentetaja. com), con sede en Madrid; la Librería Anticuaria Valdés (www.anticuaria. com), Ojanguren (www.ojanguren.net) y Cervantes (www.las.es/ cervantes) en Oviedo; o Herder (www.boks-world.com), Puvill (www.puvill.com) y La Central Llibreters (www.lacentral.com) en Barcelona; junto a otras que, pese a no estar ubicadas en grandes centros urbanos o en poblaciones de tradición universitaria, ofrecen completas bases de datos con numerosos títulos de distintas especialidades, y entre las que destacaremos, a modo de ejemplo, las librerías Robafaves (www.robafaves.com), de Mataró, y Canaima (www. libreriacanaima.com) en Las Palmas de Gran Canaria. Todas ellas cuentan con extensos catálogos en los que podemos buscar obras por el título, el autor o la materia. Varias ofrecen organizada la lista de temas, lo que facilita enormemente la búsqueda. En muchos casos también es posible seleccionar títulos y adquirirlos directamente mediante correo electrónico. En algunas de ellas, no obstante, el usuario debe solicitar previamente una clave de acceso, por teléfono o e-mail.

Junto a las librerías clásicas citadas, existen otras especializadas en ofrecer sus servicios a través de la red. Entre ellas destacan las norteamericanas Amazon (www.amazon.com), que se anuncia a sí misma como la más completa de la red, con dos millones y medio de títulos disponibles, y Barnes and Noble (www.barnesandnoble.com), con más de un millón de libros en su catálogo electrónico; las británicas Internet Book Shop (www.bookshop.co.uk) y la Bookstacks Unlimited (www.books.com); y en España, la librería Bol (www.bol.com), sección española de una librería multinacional, que cuenta con un fondo muy completo, la casa Interbook, librería electrónica sevillana (www.disbumad.es), la madrileña Libronet (www.libronet.es), la barcelonesa Libroweb (www.libroweb.com), o las librerías virtuales Mundi Prensa Libros (www.mundiprensa.es), Edera (www.edera.es), Papiros (www. papiros.es) y Díaz de Santos (www.diazdesantos.es).

La consulta de los títulos disponibles en las editoriales y librerías puede realizarse además de una manera cómoda y directa a través de algunas páginas que ofrecen la relación ordenada de sus principales datos, e incluso la posibilidad de acceder a sus páginas Web o a sus catálogos. Entre ellas destaca- 
remos el directorio de editoriales españolas del Ministerio de Educación y Cultura (www.mcu.es/pic/spain/EDIT.html), la lista de editoriales españolas elaborada por la Biblioteca Nacional, con vínculo de acceso electrónico a muchas de ellas (www.bne.es/editesp.html), y otra base de datos similar, referente a librerías, también elaborada por la Biblioteca Nacional (www.bne.es/libesp.html). Entre las entidades de ámbito internacional destacaremos la británica Bubl Link (link.bubl.ac.uk: 80/publishing), que ofrece un completo listado de editores y libreros, con datos relativos a cada uno y acceso a sus catálogos.

Dado el creciente ritmo con que la información disponible en Internet se amplia y diversifica, cualquier inventario de recursos disponibles, y por supuesto las páginas precedentes, ha de quedar anticuado en poco tiempo. Por ello es necesario mantener la información actualizada buscando recursos nuevos que aparezcan en la red, con vistas a aprovecharlos en el trabajo de investigación. Para ello resultan imprescindibles los llamados portales o buscadores, programas específicos de dominio público que nos permiten la localización de nuevas páginas y datos. Algunos de ellos presentan la información agrupada por temas y subtemas, lo cual nos facilita su rápida localización, aunque la información que conseguiremos por este método no siempre será completa. Por ello, tales programas también ofrecen la posibilidad de introducir nuestros propios criterios de búsqueda, para lo cual deberemos escribir la palabra o las palabras clave que nos interesen, completas, mediante comodines, o bien utilizando los operadores booleanos que más arriba citábamos, si el programa lo permite. Entre los buscadores más comunes destacan Altavista (www.altavista.digital.com), probablemente el más completo, y en el que podemos localizar información a través de los índices que el propio programa nos proporciona, o bien acudiendo al sistema de búsqueda simple o de búsqueda avanzada con utilización de operadores; el programa denominado Yahoo (www.yahoo.com), buscador muy popular, en que la información aparece agrupada en completos índices temáticos, o es accesible mediante los datos que el propio usuario escribe; y los buscadores Olé (www.ole.es), Ozú (www.ozu.com) y Mundo Latino (www.mundolatino.org/index.htm), similares a los anteriores, aunque especializados en páginas referentes al ámbito hispánico en general. El primero de ellos se encuentra actualmente integrado en el portal denominado Terra (www.terra.com), común a España y a varios países de Iberoamérica.

Para terminar, y como complemento de la lista de direcciones de Internet que hemos comentado en páginas precedentes, ofrecemos acerca del tema la siguiente: 
BIBLIOGRAFÍA ESENCIAL EN ESPAÑOL

Ali GaGo, Ismail; GANUZA FernánDEZ, José Luis (1996): Internet en la educación. Madrid, Anaya Multimedia, $240 \mathrm{pp}$.

ANDRIEU, Olivier (1997): Cómo buscar y encontrar en Internet, Barcelona, Gestió $2000,200 \mathrm{pp}$.

BARÓ, Jaume (1997): Internet: Manual d'ús, Barcelona, Llibres de L'Índex, 190 pp. (en catalán).

Beato Víbora, Manuel; Morera Mainar, Jorge; Ribeiro Montero, Raúl (1997): Internet, Madrid, Anaya Multimedia, $384 \mathrm{pp}$.

Blecua, José Manuel; Clavería, Gloria; Sánchez, Carlos; Torruella, Joan, eds. (1999): Filología e informática. Nuevas tecnologías en los estudios filológicos, Barcelona, Universidad Autónoma de Barcelona, 493 pp.

Bou, Enric (1997): «A la búsqueda del aura. Literatura en Internet», en ROMERA CASTillo, José; Gutiérrez Carbajo, Francisco; García-Page, Mario, eds., Literatura y multimedia, pp. 163-180.

BuENAVENTURA. Ramón (1999): Las respuestas. Todo lo que usted siempre quiso preguntar sobre Internet, Madrid, Debate, $214 \mathrm{pp}$.

Burdea, Grigore; COIFFET, Philippe (1996): Tecnologías de la realidad virtual, Barcelona, Paidós Ibérica, $432 \mathrm{pp}$.

Carballar Falcón, José Antonio (1997): Internet. Cómo descubrir el mundo, Madrid, Ra- Ma. 528 pp.

CARRo, José; Wang. Bema (1997): Guía práctica para el uso del ordenador en la creación literaria. De la pluma a la araña, Madrid, Ediciones y Talleres de Escritura Creativa Fuentetaja, 302 pp.

Casanova Seuma. Lourdes (1998): Internet para profesores de español, Madrid, Edelsa, 64 pp.

Downing, Douglas; Covington, Michael; Covington, Melody Hanldin (1997): Diccionario de términos informáticos e Internet, Madrid, Anaya Multimedia, 416 pp.

Esebbag Benchimol. Carlos; Martinez Valero, Julián (1997): Internet, Madrid, Anaya Multimedia, $352 \mathrm{pp}$.

FAINSTEIN LAMUEDRA, Graciela (1997): América Latina en Internet: Manual y fuentes de información, Madrid, CSIC, $252 \mathrm{pp}$.

FUENTES I PUJOL. Maria Eulàlia (1997): La información en Internet, Barcelona, Cims, $240 \mathrm{pp}$.

Gahete Diaz. J. Luis; García San luis, Alejandro; García Merayo, Félix (1998): Internet: Guía práctica para estudiantes, Madrid, McGraw-Hill, 96 pp.

García Marco. Francisco Javier; Tramullas Saz. Jesús (1996): World Wide Web. Fundamentos, navegación y lenguajes de la red mundial de información, Madrid, Ra-Ma, 304 pp. 
García Mongay, Fernando (1998a): Libros en Internet, Madrid, Espasa-Calpe, 133 $\mathrm{pp}$.

- (1998b), Publicar en Internet: HTML para principiantes, Madrid, Ra-Ma, $106 \mathrm{pp}$. GILSTER, Paul (1996): El nuevo navegante de Internet. Viaje por el apasionante mundo de las redes, Madrid, Anaya Multimedia, $784 \mathrm{pp}$.

González Sánchez, José Luis; Sánchez A., Marisol; Gazo Cervero. Alfonso (1998): Autopistas de la información e Internet: tecnología, servicios, peajes y normas de navegación. Cáceres, Universidad de Extremadura, 456 pp.

GraCia ARMENDÁRIZ, Juan (1998a): «Humanidades y recursos de información en Internet», El Profesional de la Información, VII, 3, pp. 20-25.

- (1998b): «Selección de fuentes de información en Internet pura el estudio de la literatura», Revista General de Información y Documentación [Escuela Universitaria de Biblioteconomía y Documentación, Universidad Complutensc, Madrid], VIII, 1, pp. 203-212.

Grupo ANONIMI Internautae (1998): Manual imprescindible de Internet, Madri: Anaya Multimedia, $386 \mathrm{pp}$.

GuIM, Jordi (1997): Internet: La guía más fácil para dominar la red, Barcelona, Gestío $2000,232 \mathrm{pp}$.

Hann, Harley (1998): Páginas de Internet 99, Madrid, McGraw-Hill, 922 pp.

- Stout, Rick (1996a): Cómo trabajar con Internet, Madrid, McGraw-Hill, 5 vols.

- (1996b), Internet: Manual de referencia, Madrid, McGraw-Hill, 692 pp.

Insa Ghisaura, Daniel; Morata Sebastián. Rosario (1998): Multimedia e Internet, Madrid, Paraninfo, 291 pp. + 1 disco CD-Rom.

Isla Montes, José Luis; Ortega Molina, Francisco Damián; Ferreiro Ramos, María José (1998): Trabajando conectados a Internet, Cádiz, Universidad de Cádiz, 114 pp.

KeAting, Victoria (1998), Literatura en Internet, Madrid, Anaya Multimedia, 190 pp. MARCos MARÍN, Francisco (1994): Informática y humanidades, Madrid, Gredos, 816 $\mathrm{pp}$.

Marcos Mora. María del Carmen (1998): «El acceso a los catálogos en línea a través de Internet», Revista Gulleral de Información y Documentación [Escuela Universitaria de Biblioteconomía y Documentación, Universidad Complutense, Madrid], VIII, 2, pp. 85-118.

MARTiNEZ LÓPEZ, Francisco J., y oti us , 1997): Internet para investigadores. Relación y localización de recursos en la red para investigadores y universitarios. Huelva, Universidad de Huelva, 2. ${ }^{a}$ edic, 128 pp. +1 disquete.

Martinez Soler, José A.; Ros, Francisco; Santillana, Ignacio (1996): Las autopistas de la información, Madrid, Debate, $121 \mathrm{pp}$.

Merlo Vega, José Antonio; Sorli Rojo. Ángela (1997): Biblioteconomía y documentación en Internet, Madrid, CSIC, 149 pp. + 1 disquete.

MESO AYERDI, KOLDO (1997): Educación en Internet, Madrid, Anaya Multimedia, 208 pp. 
Morales Barroso. José; Lázaro García, Juan Luis; Soto Araneta, Miguel (1999): Internet, Madrid, L\&M Data Communications, $201 \mathrm{pp}$.

Parra Pérez. Beatriz (1996): Internet en España, Madrid, Anaya Multimedia, 248 pp.

- (1998): Guía visual de Internet, Madrid, Anaya Multimedia, 204 pp. + 1 CDRom.

PARRA. Eduardo (1997): Diccionario de Internet, Madrid, Noesis, 184 pp.

PeN̂a Pérez. Rosario (1997): La educación en Internet: Guía para su aplicación práctica en la enseñanza, Barcelona, Inforbook's, $415 \mathrm{pp}$.

- (1998): Aprendiendo a viajar por Internet: curso práctico, Barcelona, Inforbook's, 448 pp.

Perry, Paul J. (1996): Los secretos de World Wide Web, Madrid, Anaya Multimedia, $752 \mathrm{pp}$.

RAMONET. Ignacio, ed. (1998): Internet, el mundo que llega: los nuevos caminos de la comunicación, Madrid, Alianza Editorial, $304 \mathrm{pp}$.

Romera CASTILLO. José (1997): «Literatura y nuevas tecnologías», en ROMERA CAStillo, José; Gutiérrez Carbajo. Francisco; GarCía-PAGE. Mario, eds., Literatura y multimedia (ver referencia siguiente), pp. 13- 82.

- Gutiérrez Carbajo. Francisco; García-Page. Mario, eds. (1997): Literatura y multimedia. Actas del VI Seminario Internacional del Instituto de Semiótica Literaria. Teatral y Nuevas Tecnologías de la UNED. Madrid, Visor Libros, 386 $p p$.

RUIPÉREZ. Germán (1997): «Internet como recurso multimedia del investigador de literatura", en Romera Castillo, José; Gutiérrez Carbajo, Francisco; GarcíaPAGE. Mario, eds., Literatura y multimedia, pp. 149-161.

- Mairal. Ricardo, y otros (s. f.): Aprender e investigar con Internet, Madrid, UNED, $176 \mathrm{pp}$.

Sánchez Navarro. José Daniel; Samaniego Sánchez, María José (1999): Páginas de Internet en español, 99, Madrid, McGraw-Hill, 280 pp.

Stuhrmann. Jan Christoph; FASH, Michael (1999): Las mejores direcciones Internet 99: el gran compendio, Barcelona, Marcombo, $768 \mathrm{pp}$.

SuÁrez, Joaquín María; GonzÁlez LóPEZ, Óscar R. (1997): Manual avanzado de Internet, Madrid, Anaya Multimedia, $467 \mathrm{pp}$.

Sueda, Jaume; Calvo. Aina Maria (1998): Recursos en Internet. Benissalem (Baleares), $\mathrm{Di} 7,280 \mathrm{pp}$.

UbIETO ARTUR. Antonio Paulo (1995): Documentación automatizada: manual de uso de la red Internet, Zaragoza, Anubar, $326 \mathrm{pp}$.

Zurdo. David: ACEvedo. Fernando; Sicilia. Alejandro (1998a): Buscadores de Internet, Madrid, Paraninfo, 248 pp.

- (1998b), Internet: historia, funcionamiento y estructura, conexión, servicios y programas, buscadores, seguridad, direcciones de interés, Madrid, Paraninfo, $268 \mathrm{pp}$. 